\title{
Un análisis bibliométrico en el área de la Medicina: colaboración científica entre Brasil y España (2002-2011)
}

\author{
Adolfo Alonso Arroyo * \\ Ely Francina Tannuri de Oliveira \\ Maria Cláudia Cabrini Grácio ** \\ Andrés Pandiella *** \\ Rafael Aleixandre Benavent ****
}

Artículo recibido:

19 de mayo de 2014.

Artículo aceptado:

14 de mayo de 2015.

\section{ResumeN}

Esta investigación tiene por objetivo analizar la evolución temporal de la producción científica en colaboración entre Brasil y España en el área de la Medicina, en el periodo 2002-2011, además de identificar las instituciones y países más productivos y representar y analizar las redes de colaboración institucional entre los países colaboradores. Como fuente de información se utilizó Scopus, por considerarla como la principal base de datos multidisciplinar y con mayor cobertura geográfica. Los documentos analizados se han limi-

* Universidad de Valencia, España. adolfo.alonso@uv.es

** Ambas autoras pertenecen a la Universidade Estadual Paulista "Júlio de Mesquita Filho"-UNESP-Marília, Brasil. (etannuri@marilia.unesp.br); (cabrini@marilia. unesp.br)

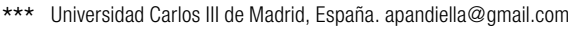

$\star \star \star \star$ Unidad de Información e Investigación Social y Sanitaria. Universidad de Valencia-CSIC. IHMC López Piñero, Valencia. España. rafael.aleixandre@uv.es 
tado a los artículos originales. Se recuperaron 1121 artículos científicos, con un total de 13906 firmas. Se calculó, para cada año, la tasa de crecimiento anual de la colaboración científica entre Brasil y España. Es significativo reseñar que han participado instituciones de 121 países diferentes, lo que demuestra el alto grado de internacionalización de los trabajos recogidos, y una red de colaboración científica en la que participan 51 países con al menos 15 contribuciones. Se constata el alto grado de colaboración entre estos dos países y el aumento significativo desarrollado a lo largo de los años en el área de la Medicina, así como su participación con otros países, si bien resulta importante resaltar que en más de la mitad de los trabajos (58 \%) se ve implicado un tercer país, impulsando la cooperación internacional.

Palabras clave: Redes de Colaboración; Colaboración Científica; Brasil; España; Medicina.

\section{Abstract}

A bibliometric analysis of collaboration between Brazil and Spain in the field of medical research from 2002 to 2011

Adolfo Alonso-Arroyo, Ely Francina-Tannuri de Oliveira, Maria Cláudia Cabrini-Grácio, Andrés Pandiella and Rafael Aleixandre-Benavent

This study analyzes the development of Spanish-Brazilian collaborative scientific production in the field of medical research between 2002 and 2011, identifying the most productive institutions, the proportion of researchers from each country and bi-lateral collaborative networks. Data were gathered from the Scopus database, which offers broad, international coverage of multidisciplinary research. A study sample of 1,121 original scientific articles signed by 13,906 researchers were retrieved, on the basis of which the annual growth rate of Spanish-Brazilian medical research was calculated. A remarkably high degree of internationalization was found, with 121 countries participating in the papers sampled. Moreover, Fully 51 countries in this international network boast at least 15 contributions. The study finds a high degree of collaboration between Spain and Brazil, and significant growth of 
collaboration in the area of medical research, including collaborations with other countries, with fully $58 \%$ of the sample involving a third country.

Keywords: Collaboration networks; scientific collaboration; Brazil; Spain; Medicine.

\section{INTRODUCCIÓN}

$\mathrm{A}_{\text {España se encontraban entre los } 11 \text { países que han experimentado una }}$ mayor tasa de crecimiento a nivel mundial en su actividad científica (Glänzel, Leta y Thijs, 2006).

La participación brasileña en la ciencia mundial durante el periodo 2002 2006 aumentó en todas las áreas de conocimiento y ocupó una posición de liderazgo entre los países de América Latina, representando aproximadamente el $50 \%$ de la producción de los países perteneciente a este grupo. Para Brasil, la medicina es una de las tres áreas con el mayor volumen de producción científica y presenta una intensa colaboración internacional con estos países, así como América del Norte y Europa, destacando España como uno de los principales socios de esta colaboración (IRD e IEDCYT-CSIC, 2009).

Consecuentemente, la producción brasileña indizada en las bases de datos internacionales ha crecido un $38.4 \%$ en el periodo, cifra bastante superior al crecimiento mundial, situado en un $19 \%$. Entre las áreas de mayor prominencia de la participación científica de este país en el ámbito internacional destacan Medicina, con un crecimiento significativo entre los países de América Latina; Botánica y Zoología (IRD e IEDCYT-CSIC, 2009; FAPESP, 2011).

Por su parte, la evolución de España en el contexto internacional muestra que la producción científica se duplica entre 2000 y 2010 al pasar de los 28062 documentos (en 2001) a los 66655 publicados en 2010. La aportación española a la producción científica mundial se ha incrementado considerablemente en los últimos años, pasando del $2.5 \%$ en 2003-2007 a $2.8 \%$ en 2006-2010. Desde 2008 la producción científica española se acerca al $3 \%$ de la producción mundial y se han diversificado cada vez más las áreas científicas en las que publica. Sin embargo, y a pesar de aumentar el volumen de producción, en 2010 España pasó de la novena a la décima posición en el ranking mundial de producción científica, debido al rápido crecimiento de los países emergentes, concretamente de India. 
España ha diversificado las áreas científicas en las que publica, sobre todo a partir de 2006, llegando a publicar en 288 categorías temáticas distintas en 2010. Destaca, como en años anteriores, y muy por encima del resto de áreas, Medicina, con un $21.4 \%$ del total de la producción científica española en 2010. Le siguen áreas como Agricultura y Ciencias Biológicas (8.8 \%), Bioquímica y Biología Molecular (8.3 \%), Química (6.8 \%) y Psicología (6.6 \%) (Moya-Anegón, 2013).

Según la base de datos Scopus y asociadas a las cuestiones citadas, Brasil y España se sitúan entre los principales países productores de Iberoamérica en el área de Medicina, con las mejores posiciones en el ranking mundial del área durante el periodo 1996-2011 (Elsevier B.V. 2014). En este contexto, la tasa de crecimiento de la producción científica en colaboración entre Brasil y España, en general, fue mayor que la tasa de crecimiento científico de estos países separadamente en el periodo estudiado, como puede ser confirmado a partir de los datos de SCImago (2013).

La colaboración científica entre países ha consolidado la internacionalización del conocimiento nuevo y de la ciencia producida (Glänzel, 2003). Este autor estudió la relación entre la productividad y la colaboración científica, demostrando que ambas están relacionadas, especialmente en algunas áreas, como la Biomedicina y la Química.

Según los estudiosos que buscan evaluar la producción científica mundial, los trabajos con colaboración internacional tienen mayor impacto y visibilidad en la comunidad científica, lo que motiva a los gobiernos a proponer iniciativas en el sentido de mejorar el comportamiento colaborativo de sus investigadores (Glänzel y Lange, 2002; Persson, Glänzel y Dannell, 2004; Iribarren-Maestro, Lascurain-Sánchez y Sanz-Casado, 2009).

En un nivel extramural, principalmente entre los países, la colaboración científica se ha convertido en una práctica imprescindible para alcanzar la masa crítica que consolide y posibilite la internacionalización de conocimiento nuevo y el análisis de la ciencia producida (Katz y Martin, 1997; Glänzel, 2003).

La colaboración científica entre autores, instituciones o países refleja un rol de intercambio de ideas, en el que se identifican un conjunto de objetivos centrales de un proyecto, lo que supone una división en el trabajo e interacción entre los investigadores, así como una comunicación fluida de información, ampliando la posibilidad de establecer nuevos enfoques y herramientas que permitan promover una red en la que se relacionen los colaboradores (Balancieri et al., 2005; Olmeda-Gómez, Perianes-Rodríguez y Ovalle-Perandones, 2008). 
Un indicador de la actividad de la colaboración científica es la coautoría. Entre sus ventajas destacan que se constituye con datos objetivos, pudiendo ser ratificada o comprobada por estudios de otros investigadores; representa una metodología accesible y amigable para cuantificar la colaboración; posibilita trabajar con grandes universos que conducen a resultados estadísticamente más significativos que en aquellos donde se utilizan "estudios de casos" (Katz y Martin, 1997). El análisis de coautoría refleja un papel posible de intercambios entre los investigadores y constituye un procedimiento significativo, medido por el número de publicaciones en colaboración entre autores, instituciones o países y utilizado para identificar y mapear la cooperación regional, nacional o internacional. Por lo tanto, el análisis de las coautorías posibilita describir e incorporar la estructura de un grupo que pueda ser representada por una red social. Según Wasserman y Faust (1994), el término 'red social' se refiere al conjunto de autores y las relaciones existentes entre ellos. El análisis de redes tiene como objetivo modelar las relaciones entre los autores, a fin de retratar, describir y representar la estructura de un grupo.

Según Otte y Rousseau (2002), el análisis de redes sociales (ARS) es un procedimiento interdisciplinario para la investigación de la estructura social y destacan que un ARS enfatiza las relaciones entre los autores; sin embargo, tanto los vínculos relacionales como las características individuales son necesarias para un completo entendimiento de un fenómeno social. En bibliometría los investigadores estudian las redes de colaboración, citas y otras formas de interacción social para ser implementadas y visualizadas a través de una representación gráfica. Estos estudios se agrupan en un amplio conjunto de indicadores que pueden ser clasificados en indicadores de producción, indicadores de citación e impacto e indicadores de relación (Narin, Olivastro y Stevens, 1994; Callon, Courtial y Penan, 1995; Okubo, 1997; Spinak, 1998).

Los indicadores de producción están basados en la frecuencia de las publicaciones de un investigador, grupo de investigadores, instituciones o países y tienen como objetivo reflejar su inserción en la comunidad científica, evidenciando aquellos más productivos así como las temáticas más destacadas de un área de conocimiento y las revistas principales en las cuales esta producción se incluye.

Los indicadores de relación, basados en las autorías o en las citaciones conjuntas, entre otros, son utilizados en la construcción y visualización de la colaboración científica, así como de las redes de cocitación entre los investigadores, instituciones o países, por medio de la confluencia de técnicas de análisis estadística, matemática o computacional. En esta investigación se utilizan los indicadores de producción y relación mediante el análisis de la colaboración científica. 
Es necesario subrayar la importancia que han adquirido en los últimos años los estudios sobre la evaluación de la ciencia en la Medicina, teniendo en cuenta la historia del desarrollo científico, el gran incentivo por parte de los organismos de financiación, la velocidad de producción respecto al gran volumen de literatura científica y la consolidación del área médica en el mundo científico.

Esta investigación tiene por objetivo analizar la evolución temporal de la producción científica en colaboración entre Brasil y España en el área de la Medicina en el periodo 2002-2011, además de identificar las instituciones y países más productivos y representar y analizar las redes de colaboración institucional entre los países colaboradores. Buscamos contribuir a visualizar la evolución de la colaboración científica en Medicina entre Brasil y España, destacando las principales instituciones y países que contribuyen a la consolidación de la red científica de colaboración en el área, así como al análisis de las principales temáticas estudiadas en la investigación científica desarrolladas por los investigadores de ambos países. Ello nos proporcionará una importante actualización en el estado del arte del área estudiada en los países iberoamericanos, pudiendo contribuir en la toma de decisiones en materia de política científica.

\section{Métodos}

Los registros que componen el corpus de estudio fueron descargados de la base de datos Sciverse Scopus el 21 de octubre de 2012. La estrategia de búsqueda se construyó teniendo en cuenta que debían participar como responsables de las publicaciones al menos una institución española (spain OR espanha OR España) y otra brasileña (brasil OR brazil). Además, para que un trabajo formara parte de este estudio tenían que darse las siguientes condiciones: que fueran artículos originales, publicados entre la década 2002-2011 y que estuvieran incluidos dentro del área temática de la Medicina.

Los 1121 artículos recuperados fueron importados a Bibliométricos, una base de datos relacional diseñada ad hoc utilizando el software Microsoft Access. Desde ahí se realizaron los procesos de normalización de los registros importados para la extracción de los resultados.

Los nombres de las instituciones fueron normalizados hasta el nivel de macroinstitución (universidad, hospital o complejo hospitalario, empresa, etc.), unificando las firmas en las que una misma institución firmaba de dos o más formas diferentes y así determinar el número de documentos firmados en colaboración institucional. En aquellos artículos en los que un autor fir- 
maba vinculado a más de una adscripción institucional se procedió a asignar tantas firmas como macroinstituciones se pudieran individualizar con el fin de no desestimar la productividad científica de dichas instituciones.

Para evaluar la evolución porcentual de la producción científica anual general y colaborativa entre los dos países analizados, se utilizó una tasa de crecimiento anual $T_{C}\left(X_{t}\right)$, calculada por la fórmula

$$
T_{C}\left(X_{t}\right)=\frac{X_{t}-X_{t-1}}{X_{t-1}} * 100
$$

donde $T_{C}\left(X_{t}\right)$ es la tasa de crecimiento de la producción científica en el año $t$; $X_{t}$ es la producción científica en el año $t$ y $X_{t-1}$ es la producción científica en el año $t-1$.

El análisis de las áreas temáticas se realizó mediante la identificación de las revistas nucleares disponibles a través del portal Scimago Journal \& Country Rank creado por Elsevier B.V. (SCImago, 2013).

Para tratar toda la información y calcular los indicadores bibliométricos y de redes sociales, así como la construcción de las representaciones gráficas de los clústeres de instituciones y países, se utilizó el software de código abierto de análisis y visualización de redes Pajek (Batagelj y Mrvar, 2008).

\section{Resultados}

Se presenta en la Tabla 1 la variación anual del número de artículos producidos en colaboración entre Brasil y España, el porcentaje de cada año y la tasa de crecimiento anual. Podemos observar que el número absoluto de artículos publicados en colaboración siempre es creciente, finalizando en el año 2011 con una producción seis veces mayor que al inicio del periodo (2002). Se destaca que en los últimos 3 años del periodo se concentra más del $50 \%$ del total de la producción colaborativa entre estos países.

Tabla 1. Evolución temporal de la producción colaborativa entre Brasil y España (2002-2011)

\begin{tabular}{|c|c|c|c|c|c|c|}
\hline Año & $\begin{array}{c}N^{0} \text { de art. col. } \\
\text { Bry Esp } \\
\text { Medicina }\end{array}$ & $\%$ & $\begin{array}{c}\text { TCC } \\
\text { Bry Esp } \\
\text { Medicina }\end{array}$ & $\begin{array}{c}\text { TCC } \\
\text { Bry Esp } \\
\text { (general) }\end{array}$ & $\begin{array}{c}\text { Tasa } \\
\text { crecimiento Br } \\
\text { (general) }\end{array}$ & $\begin{array}{c}\text { Tasa crecimiento } \\
\text { Esp (general) }\end{array}$ \\
\hline 2002 & 33 & $2,90 \%$ & - & 195 & 13.210 & 23.973 \\
\hline 2003 & 45 & $4,00 \%$ & $36,40 \%$ & $219(12 \%)$ & $14.253(8 \%)$ & $25.887(8 \%)$ \\
\hline 2004 & 51 & $4,60 \%$ & $13,30 \%$ & $264(21 \%)$ & $16.085(13 \%)$ & $27.803(7 \%)$ \\
\hline 2005 & 64 & $5,70 \%$ & $25,50 \%$ & $292(11 \%)$ & $17.470(9 \%)$ & $29.851(7 \%)$ \\
\hline
\end{tabular}




\begin{tabular}{|c|c|c|c|c|c|c|}
\hline 2006 & 85 & $7,60 \%$ & $32,80 \%$ & $439(50 \%)$ & $24.636(41 \%)$ & $35.184(18 \%)$ \\
\hline 2007 & 102 & $9,10 \%$ & $20,00 \%$ & $484(10 \%)$ & $27.572(12 \%)$ & $38.443(9 \%)$ \\
\hline 2008 & 137 & $12,20 \%$ & $34,30 \%$ & $589(22 \%)$ & $31.016(12 \%)$ & $41.168(7 \%)$ \\
\hline 2009 & 161 & $14,40 \%$ & $17,50 \%$ & $626(6 \%)$ & $34.107(10 \%)$ & $44.575(8 \%)$ \\
\hline 2010 & 222 & $19,80 \%$ & $37,90 \%$ & $812(30 \%)$ & $37.311(9 \%)$ & $47.923(8 \%)$ \\
\hline 2011 & 221 & $19,70 \%$ & $-0,50 \%$ & $978(20 \%)$ & $40.480(8 \%)$ & $52.367(9 \%)$ \\
\hline Total & 1.121 & $100,00 \%$ & & 4.891 & 256.130 & 367.174 \\
\hline $\begin{array}{c}\text { Tasa } \\
\text { Crecimiento } \\
\text { 2002-2011 }\end{array}$ & & & $569,69 \%$ & $401,00 \%$ & $206,43 \%$ & $118,44 \%$ \\
\hline
\end{tabular}

* TCC = Tasa crecimiento colaborativo; $\mathrm{Br}=$ Brasil; Esp = España

La tasa de crecimiento de colaboración entre Brasil y España durante todos los años se presenta de manera positiva y por encima de la tasa de crecimiento de la producción total de estos países, sobre todo en los años 2003, 2004, 2006, 2010 y 2011, con excepción de los años 2007 y 2009 en los que se invierte esta relación. Si se analiza la tasa de crecimiento para cada uno de los países, se observa cómo la producción científica brasileña ha estado por encima de la tasa de crecimiento de la española durante todo el periodo, a excepción del año 2011 y especialmente en 2010, año en el que el porcentaje de crecimiento de la colaboración es al menos tres veces mayor que la tasa de crecimiento de la producción en estos países.

De los 1121 artículos analizados, 474 (42.2\%) fueron escritos exclusivamente entre Brasil y España y, de ellos, 204 artículos (43\%) fueron indexados únicamente en el campo de la Medicina, sin interactuar con otras áreas afines.

La colaboración científica cada vez está más extendida, si bien puede variar dependiendo de las áreas del conocimiento. En nuestro estudio, esta colaboración está latente en el $100 \%$ de los trabajos ya que partimos de la premisa de que fueron firmados por al menos un autor/a español/a y otro/a brasileño/a.

Nuestro estudio tenía como premisa que la muestra contara con la participación al menos de un investigador español y otro brasileño, por lo que el $100 \%$ de los trabajos han sido firmados por al menos 2 autores. Los 1121 artículos tuvieron un total de 13906 firmas, destacando el mayor porcentaje $(10.44 \%)$ en los artículos firmados por 5 autores, seguido de los firmados por 6 y 7 autores con un $9.55 \%$. En la Tabla 2 se presenta la colaboración de trabajos según el número de autores, agrupando aquellos artículos firmados por un número excesivo de autores. Destacar que existen 4 artículos firmados por 246, 149, 129 y 115 autores. 
Tabla 2. Numero de autores firmantes por trabajos (2002-2011)

\begin{tabular}{|c|c|c|c|c|}
\hline $\mathrm{N}^{0}$ firmas / trabajo & Trabajos & $\%$ trabajos & Total firmas & $\%$ firmas \\
\hline $1^{*}$ & 8 & $0,71 \%$ & 8 & $0,06 \%$ \\
\hline 2 & 34 & $3,03 \%$ & 68 & $0,49 \%$ \\
\hline 3 & 53 & $4,73 \%$ & 159 & $1,14 \%$ \\
\hline 4 & 74 & $6,60 \%$ & 296 & $2,13 \%$ \\
\hline 5 & 117 & $10,44 \%$ & 585 & $4,21 \%$ \\
\hline 6 & 107 & $9,55 \%$ & 642 & $4,62 \%$ \\
\hline 7 & 107 & $9,55 \%$ & 749 & $5,39 \%$ \\
\hline 8 & 88 & $7,85 \%$ & 704 & $5,06 \%$ \\
\hline 9 & 74 & $6,60 \%$ & 666 & $4,79 \%$ \\
\hline 10 & 64 & $5,71 \%$ & 640 & $4,60 \%$ \\
\hline 11 & 40 & $3,57 \%$ & 440 & $3,16 \%$ \\
\hline 12 & 33 & $2,94 \%$ & 396 & $2,85 \%$ \\
\hline 13 & 32 & $2,85 \%$ & 416 & $2,99 \%$ \\
\hline 14 & 21 & $1,87 \%$ & 294 & $2,11 \%$ \\
\hline 15 & 21 & $1,87 \%$ & 315 & $2,27 \%$ \\
\hline 16 & 13 & $1,16 \%$ & 208 & $1,50 \%$ \\
\hline 17 & 12 & $1,07 \%$ & 204 & $1,47 \%$ \\
\hline 18 & 20 & $1,78 \%$ & 360 & $2,59 \%$ \\
\hline 19 & 17 & $1,52 \%$ & 323 & $2,32 \%$ \\
\hline 20 & 17 & $1,52 \%$ & 340 & $2,44 \%$ \\
\hline 21 & 14 & $1,25 \%$ & 294 & $2,11 \%$ \\
\hline 22 & 13 & $1,16 \%$ & 286 & $2,06 \%$ \\
\hline 23 & 8 & $0,71 \%$ & 184 & $1,32 \%$ \\
\hline 24 & 11 & $0,98 \%$ & 264 & $1,90 \%$ \\
\hline 25 & 9 & $0,80 \%$ & 225 & $1,62 \%$ \\
\hline 26 & 4 & $0,36 \%$ & 104 & $0,75 \%$ \\
\hline 27 & 6 & $0,54 \%$ & 162 & $1,16 \%$ \\
\hline 28 & 9 & $0,80 \%$ & 252 & $1,81 \%$ \\
\hline 29 & 12 & $1,07 \%$ & 348 & $2,50 \%$ \\
\hline 30 & 9 & $0,80 \%$ & 270 & $1,94 \%$ \\
\hline $31-50$ & 55 & $4,91 \%$ & 2.103 & $15,12 \%$ \\
\hline $51-100$ & 15 & $1,34 \%$ & 962 & $6,92 \%$ \\
\hline$>100$ & 4 & $0,36 \%$ & 639 & $4,60 \%$ \\
\hline Total & 1.121 & $100,00 \%$ & 13.906 & $100,00 \%$ \\
\hline
\end{tabular}

El índice de colaboración durante la década estudiada es de 12.4 autores por trabajo. En relación con la evolución del índice de colaboración a lo largo del periodo estudiado, éste se mantiene entre los 9 y 12 autores por trabajo en los 8 primeros años de estudio, aumentando en los dos últimos años hasta un 13.72 para el año 2010 y de un 15.09 en el año 2011. Hay que tener en 
cuenta que los trabajos con un mayor número de firmas se sitúan en estos dos últimos años.

Los artículos en colaboración entre Brasil y España fueron publicados en 581 revistas diferentes. Entre las áreas más productivas destacan Inmunología (92 revistas), Medicina General (73), Oncología (48), Endocrinología (38) o Neurología y Salud Pública, Medio Ambiente y Salud Ocupacional con 35 revistas. Las revistas que han publicado un mayor número de artículos son Plos One y New England Journal of Medicine con 18 trabajos; Annals of the Rheumatic Diseases y Journal of Clinical Microbiology con 12 y Arquivos de Neuro-Psiquiatria con 10. Asimismo, si analizamos el país de publicación de las revistas donde publican los investigadores brasileños y españoles y lo contrastamos con el total de artículos publicados en dichas revistas, se constata que los autores publican principalmente en revistas americanas o británicas antes de publicar en las revistas de su país de origen. Reino Unido participa con 179 revistas (31 \%) y 307 trabajos (27,4 \%); Estados Unidos aporta 178 revistas $(30 \%$ ) pero con un mayor número de artículos 389 (34.7\%). Por su parte, España presenta 49 revistas $(8.4 \%$ ) y 102 artículos (9\%) y Brasil 43 revistas $(7.4 \%)$ pero con 112 trabajos (10\%). El $23 \%$ de revistas restante se reparte entre otros 24 países, con 211 trabajos (18.9\%).

La colaboración institucional se aglutina en 2897 instituciones diferentes, de las cuales en cada documento al menos firma una institución española y otra brasileña. Hay 178 documentos $(15.88 \%$ ) que han sido firmados por dos instituciones solamente (una de cada país, España y Brasil), mientras que 212 han sido firmados por tres (18.91\%) y 151 trabajos por cuatro instituciones $(13.74 \%)$. En el lado opuesto, se sitúan un artículo firmado por hasta 138 instituciones diferentes.

Si el cálculo de los trabajos se realiza teniendo en cuenta los países de afiliación de las instituciones los datos varían: 484 artículos son firmados por dos países, que en este caso serán Brasil y España, lo que supone un 43.18 \% del total de trabajos; por tres países hay 161 documentos $(14.36 \%$ ) y así va descendiendo hasta encontrarnos en el otro polo, un trabajo realizado por hasta 42 países diferentes.

De las 2897 instituciones que firman los documentos recuperados, 411 son instituciones españolas y 299 brasileñas. En la Tabla 3 se presenta la productividad de las instituciones con más de 20 documentos publicados desglosados por países. Destacan dos instituciones con más de 100 documentos: la institución brasileña Universidade de São Paulo (USP) con 222 trabajos, más del doble que la segunda institución más productiva, y en segundo lugar el Hospital Clínic i Provincial de Barcelona con 102 trabajos. Entre las instituciones de otros países destacan la Universidad de Buenos Aires con 48 traba- 
jos y la Universiy of Toronto de Canadá con 36 documentos. Como se puede apreciar hay un ligero predominio de universidades, que se completan con la participación de hospitales o centros sanitarios e institutos de investigación.

Tabla 3. Instituciones más productivas por país (España, Brasil y otros) con más de 20 documentos (2002-2011)

\begin{tabular}{|c|c|c|}
\hline País & Institución & $\mathrm{N}^{0} \mathrm{Doc}$. \\
\hline \multirow{13}{*}{ Brasil } & Universidade de São Paulo (USP) & 222 \\
\hline & Fundação Oswaldo Cruz (FIOCRUZ) & 97 \\
\hline & Universidade Federal de São Paulo (UNIFESP) & 95 \\
\hline & Universidade Federal do Rio de Janeiro (UFRJ) & 94 \\
\hline & Universidade Federal do Rio Grande do Sul (UFRGS) & 82 \\
\hline & Hospital de Clínicas de Porto Alegre (HCPA) & 79 \\
\hline & Universidade Estadual de Campinas (UNICAMP) & 51 \\
\hline & Universidade Estadual Paulista (UNESP) & 47 \\
\hline & $\begin{array}{l}\text { Hospital das Clínicas da Faculdade de Medicina da Universidade de São Paulo } \\
\text { (HCFMUSP) }\end{array}$ & 47 \\
\hline & Universidade Federal de Minas Gerais (UFMG) & 44 \\
\hline & Universidade Federal de Pelotas (UFPEL) & 24 \\
\hline & Santa Casa de Misericórdia de Porto Alegre & 24 \\
\hline & Pontifícia Universidade Católica Do Rio Grande Do Sul (PUCRS) & 21 \\
\hline \multirow{24}{*}{ España } & Hospital Clínic i Provincial de Barcelona & 102 \\
\hline & Universitat de Barcelona & 92 \\
\hline & Universitat Autònoma de Barcelona & 62 \\
\hline & Instituto de Salud Carlos III & 54 \\
\hline & Universidad Complutense de Madrid & 51 \\
\hline & Complejo Universitario La Paz & 51 \\
\hline & Universidad Autónoma de Madrid & 45 \\
\hline & Hospitals Vall d'Hebron & 40 \\
\hline & Universitat de València & 39 \\
\hline & Universidad de Granada & 38 \\
\hline & Universidad de Santiago de Compostela & 37 \\
\hline & Universitat Rovira i Virgili & 37 \\
\hline & Institut Català d'Oncologia (ICO) & 35 \\
\hline & Institut d'Investigacions Biomèdiques August Pi i Sunyer (IDIBAPS) & 31 \\
\hline & Hospital Ramón y Cajal & 27 \\
\hline & Hospital de la Santa Creu i Sant Pau & 26 \\
\hline & Complejo Univesitario de San Carlos & 25 \\
\hline & Hospital General Universitario Gregorio Marañón & 24 \\
\hline & Universidad de León & 23 \\
\hline & Universitat Pompeu Fabra & 23 \\
\hline & Universidad de Salamanca & 22 \\
\hline & Hospital de Sant Joan de Déu & 22 \\
\hline & Hospital Universitari de Bellvitge & 22 \\
\hline & $\begin{array}{l}\text { Consorcio de Investigación Biomédica de Epidemiología y Salud Pública (CIBE- } \\
\text { RESP) de Barcelona }\end{array}$ & 22 \\
\hline
\end{tabular}




\begin{tabular}{|c|c|c|}
\hline Argentina & Universidad de Buenos Aires (UBA) & 48 \\
\hline Canadá & University of Toronto & 36 \\
\hline Austria & Medical University of Vienna & 32 \\
\hline Bélgica & University Hospitals Leuven & 30 \\
\hline $\begin{array}{l}\text { Estados } \\
\text { Unidos }\end{array}$ & Duke University Medical Center & 30 \\
\hline Francia & Université Paris V René Descartes & 29 \\
\hline Francia & Institut National de la Santé et de la Recherche Médicale (INSERM) & 28 \\
\hline Alemania & Charité - University Hospital Berlin & 26 \\
\hline $\begin{array}{l}\text { Estados } \\
\text { Unidos }\end{array}$ & University of North Carolina & 26 \\
\hline Suecia & Karolinska Institutet & 26 \\
\hline Italia & Università degli Studi di Padova & 25 \\
\hline $\begin{array}{l}\text { Estados } \\
\text { Unidos }\end{array}$ & University of Michigan & 24 \\
\hline $\begin{array}{l}\text { Estados } \\
\text { Unidos }\end{array}$ & Harvard Medical School & 24 \\
\hline Portugal & Instituto de Patología e Inmunología Molecular (IPATIMUP) & 24 \\
\hline Francia & International Agency for Research on Cancer (IARC) & 23 \\
\hline Australia & University of Melbourne & 22 \\
\hline Bélgica & Université Catholique de Louvain & 22 \\
\hline Italia & Università degli Studi di Milano & 22 \\
\hline $\begin{array}{l}\text { Países } \\
\text { Bajos }\end{array}$ & University Medical Center Groningen & 21 \\
\hline $\begin{array}{l}\text { Reino } \\
\text { Unido }\end{array}$ & University College London & 21 \\
\hline
\end{tabular}

La Figura 1 representa la red de la participación institucional, en la cual se puede identificar, aplicando un umbral de 10 o más trabajos firmados en colaboración, un gran núcleo o red de relaciones de colaboración institucional que integra 64 instituciones vinculadas entre sí.

En esta compleja red se aprecia el papel central que adquiere la Universidade de São Paulo (USP), ya que de ella salen todas las ramificaciones hacia el resto de instituciones. Se observa la participación de instituciones brasileñas (10, indicadas por cuadrados) y españolas (17, por triángulos), que colaboran junto con instituciones de otros 16 países diferentes. Se pueden destacar instituciones como la Universidad de Buenos Aires (UBA); el Instituto Catalán de Oncología (ICO); el Hospital Clínic i Provincial de Barcelona; las universidades federales de São Paulo y de Rio de Janeiro y la Fundação Oswaldo Cruz (Fiocruz), que establecen la vinculación entre la Universidade de São Paulo y el resto de instituciones participantes. 


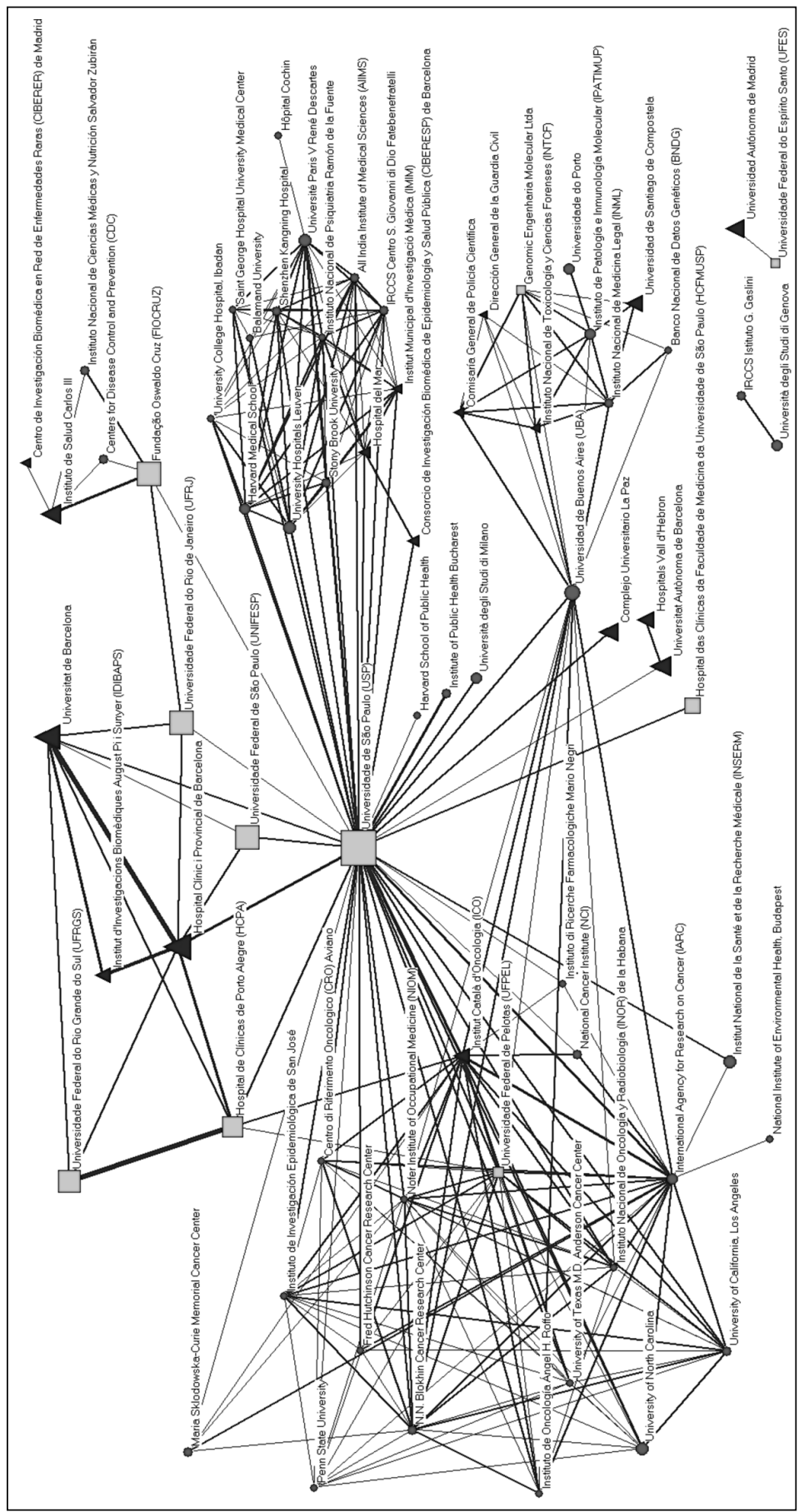

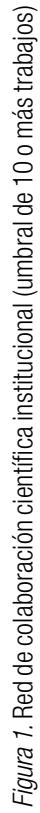


Las colaboraciones más intensas en esta red las han establecido el Hospital de Clínicas de Porto Alegre (HCPA) y la Universidad Federal do Rio Grade do Sul (UFRGS) ( $\mathrm{n}=40$ trabajos firmados conjuntamente); el Hospital Clínic i Provincial de Barcelona, por un lado con la Universitat de Barcelona $(\mathrm{n}=39)$ y por otro con el Institut d'Investigacions Biomèdiques August Pi i Sunyer (IDIBAPS) ( $\mathrm{n}=27)$; la Fundação Oswaldo Cruz (FIOCRUZ) y el Instituto de Salud Carlos III ( $\mathrm{n}=22$ ), y de nuevo el Hospital Clínic i Provincial de Barcelona, pero en este caso con un hospital brasileño, el Hospital de Clínicas de Porto Alegre (HCPA) $(\mathrm{n}=21)$.

Al pie de la Figura 1 se observan dos núcleos aislados de instituciones formados por la Universidad Autónoma de Madrid y la Universidad Federal do Espírito Santo (UFES) y por dos instituciones italianas, la Universitá degli Studi di Genova y el IRCCS Istituto G. Gaslini.

La distribución de documentos por países que colaboran con las instituciones españolas y brasileñas se aprecia en la Tabla 4. Las 2897 instituciones diferentes han aparecido en 5994 ocasiones y corresponden a 121 países diferentes de los cinco continentes. El país con el que se ha publicado un mayor número de documentos ha sido Estados Unidos, con 357 trabajos y 389 instituciones diferentes, seguido de Francia con 231 documentos y 117 instituciones, y otros como Italia, Reino Unido, Alemania y Argentina. España ha aportado al estudio 411 instituciones diferentes, mientras que Brasil lo ha hecho con 299 instituciones.

Tabla 4. Número de documentos y de instituciones por países (2002-2011)

\begin{tabular}{|l|c|c|l|c|c|}
\hline \multicolumn{1}{|c|}{ País } & $\mathbf{N}^{0}$ Inst. & $\mathbf{N}^{0}$ Doc & \multicolumn{1}{c|}{ País } & $\mathbf{N}^{0}$ Inst. & $\mathbf{N}^{0}$ Doc \\
\hline Albania & 2 & 1 & Israel & 18 & 73 \\
\hline Alemania & 115 & 191 & Italia & 141 & 206 \\
\hline Angola & 1 & 1 & Japón & 66 & 59 \\
\hline Arabia Saudita & 4 & 8 & Kenia & 2 & 3 \\
\hline Argentina & 1 & 1 & Kirguistán & 1 & 1 \\
\hline Argelia & 2 & 4 & Kuwait & 1 & 1 \\
\hline Argentina & 79 & 155 & Letonia & 5 & 14 \\
\hline Australia & 60 & 100 & Líbano & 6 & 22 \\
\hline Austria & 18 & 59 & Lituania & 5 & 11 \\
\hline Bangladesh & 2 & 2 & Luxemburgo & 3 & 4 \\
\hline Bélgica & 29 & 96 & Madagascar & 1 & 2 \\
\hline Bolivia & 10 & 11 & Malasia & 4 & 5 \\
\hline Bosnia y Herzegovina & 1 & 1 & Malawi & 2 & 2 \\
\hline Botswana & 1 & 1 & Mali & 1 & 1 \\
\hline Brasil & 299 & 1.121 & Malta & 2 & 2 \\
\hline Bulgaria & 9 & 23 & Marruecos & 6 & 6 \\
\hline Burkina Faso & 1 & 1 & México & 66 & 117 \\
\hline
\end{tabular}




\begin{tabular}{|c|c|c|c|c|c|}
\hline Camboya & 1 & 1 & Mozambique & 2 & 2 \\
\hline Camerún & 1 & 1 & Nepal & 1 & 1 \\
\hline Canadá & 88 & 148 & Nicaragua & 1 & 1 \\
\hline Chile & 23 & 48 & Nigeria & 5 & 17 \\
\hline Chipre & 1 & 1 & Noruega & 26 & 41 \\
\hline Colombia & 41 & 79 & Nueva Zelanda & 10 & 27 \\
\hline Congo & 1 & 1 & Países Bajos & 31 & 124 \\
\hline Corea del Sur & 17 & 20 & Pakistán & 1 & 1 \\
\hline Costa Rica & 12 & 38 & Palestina & 1 & 1 \\
\hline Croacia & 10 & 18 & Panamá & 7 & 6 \\
\hline Cuba & 14 & 25 & Papúa Nueva Guinea & 1 & 1 \\
\hline Dinamarca & 17 & 45 & Paraguay & 9 & 11 \\
\hline Ecuador & 16 & 18 & Perú & 25 & 40 \\
\hline Egipto & 6 & 6 & Polonia & 25 & 65 \\
\hline El Salvador & 5 & 4 & Portugal & 35 & 70 \\
\hline Emiratos Árabes Unidos & 6 & 8 & Puerto Rico & 5 & 10 \\
\hline Eslovaquia & 12 & 29 & Qatar & 1 & 1 \\
\hline Eslovenia & 2 & 7 & Reino Unido & 129 & 197 \\
\hline España & 411 & 1.121 & República Checa & 15 & 40 \\
\hline Estados Unidos & 389 & 357 & República del Chad & 1 & 1 \\
\hline Estonia & 5 & 9 & República Dominicana & 4 & 4 \\
\hline Etiopía & 2 & 2 & República Popular de China & 20 & 44 \\
\hline Filipinas & 3 & 8 & Rumanía & 12 & 42 \\
\hline Finlandia & 28 & 49 & Rusia & 14 & 38 \\
\hline Francia & 117 & 231 & Senegal & 1 & 1 \\
\hline Gambia & 1 & 2 & Serbia y Montenegro & 8 & 13 \\
\hline Georgia & 2 & 2 & Singapur & 9 & 22 \\
\hline Ghana & 2 & 2 & Siria & 1 & 1 \\
\hline Grecia & 25 & 45 & Sudáfrica & 18 & 44 \\
\hline Guadalupe & 1 & 3 & Suecia & 20 & 67 \\
\hline Guatemala & 8 & 7 & Suiza & 30 & 90 \\
\hline Guayana Francesa & 2 & 3 & Tailandia & 10 & 20 \\
\hline Guyana & 1 & 1 & Taiwán & 15 & 22 \\
\hline Haití & 1 & 1 & Tanzania & 4 & 3 \\
\hline Honduras & 4 & 7 & Túnez & 5 & 5 \\
\hline Hong Kong & 5 & 28 & Turquía & 20 & 34 \\
\hline Hungría & 21 & 46 & Ucrania & 5 & 9 \\
\hline India & 35 & 52 & Uganda & 1 & 2 \\
\hline Indonesia & 2 & 1 & Uruguay & 16 & 27 \\
\hline Irán & 7 & 5 & Venezuela & 22 & 33 \\
\hline Iraq & 1 & 2 & \begin{tabular}{|l|} 
Vietnam \\
\end{tabular} & 2 & 2 \\
\hline Irlanda & 13 & 22 & Zambia & 1 & 1 \\
\hline Islandia & 2 & 8 & Zimbabue & 2 & 2 \\
\hline Islas Feroe & 1 & 1 & & & \\
\hline
\end{tabular}




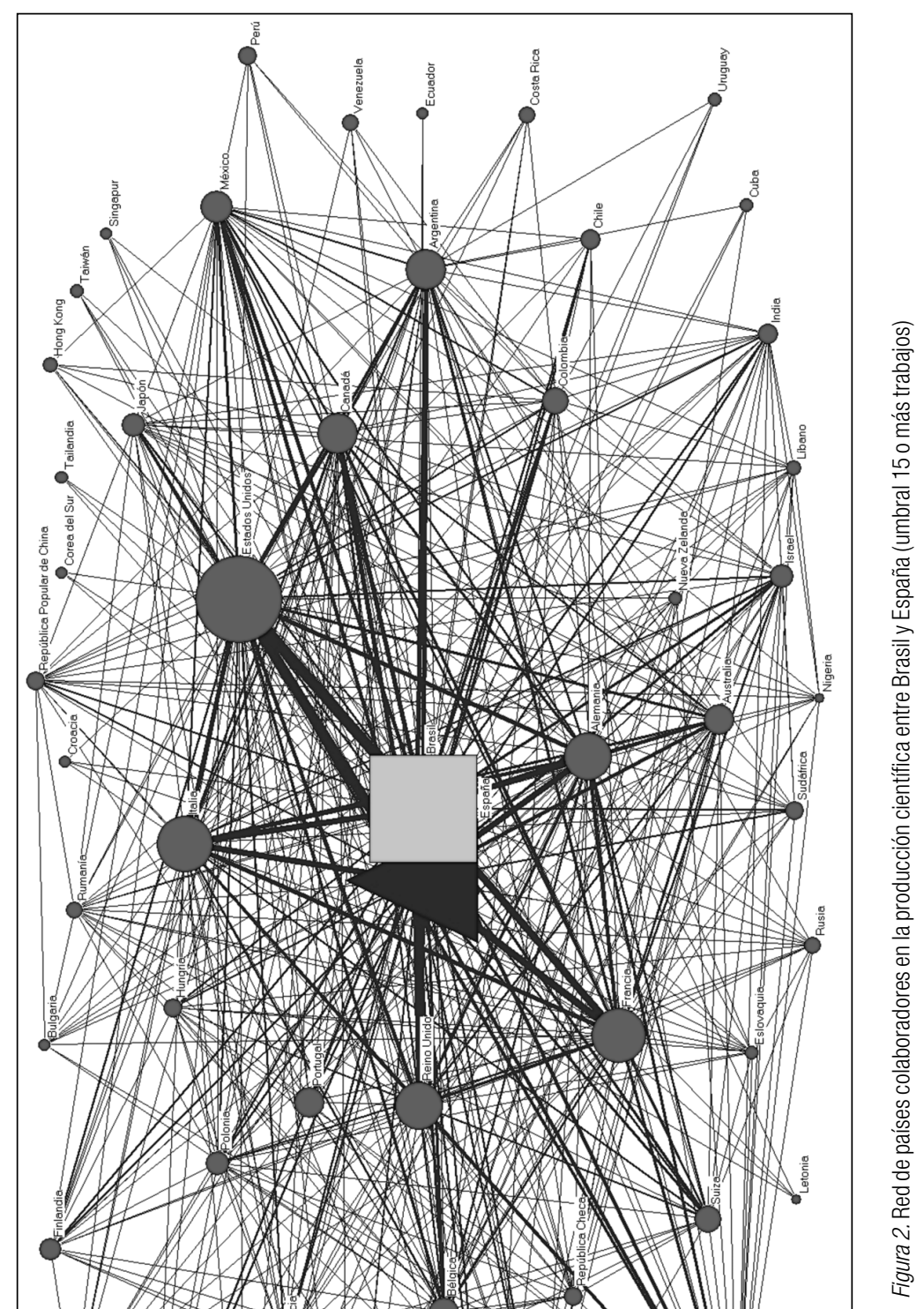


En la Figura 2 se presenta la red de colaboración entre los países de procedencia de las instituciones con un umbral de 15 o más artículos firmados en colaboración. Puesto que el estudio recoge la colaboración entre Brasil y España, se sitúan en la parte central estos países ya que colaboran en los 1121 trabajos analizados. Junto a estos países, se representan 51 más con al menos una participación de 15 trabajos en colaboración.

Destacan las grandes potencias de la investigación científica de los cinco continentes, como Estados Unidos, Canadá, Japón, China, Australia, Nueva Zelanda, Alemania, Italia, Reino Unido, Francia, India y México. También se aprecia la vinculación de proximidad en la participación de 10 países de Sudamérica y Centroamérica y 22 de la Unión Europea. Estos datos reflejan el alto grado de colaboración, siempre en aumento y la internacionalización desarrollada por Brasil y España en el ámbito de las Ciencias de la Salud.

\section{Discusión}

Este trabajo ha permitido identificar y analizar la colaboración científica entre Brasil y España, integrando el análisis bibliométrico con el análisis de redes sociales. Uno de los resultados más llamativos encontrados es que la colaboración científica entre ambos países ha aumentado durante el periodo analizado, septuplicándose entre los años 2002 y 2011. Además, la tasa de crecimiento de los trabajos en colaboración fue mayor que la tasa de crecimiento de estos países por separado.

Algunos análisis previos han mostrado como área más productiva, entre los perfiles de especialización temática en publicaciones de los países latinoamericanos, la Medicina Clínica, y casi una cuarta parte de los documentos publicados por estos países pertenecen a esta área (De Filippo y Gómez, 2011). El crecimiento individual de Brasil está en la línea con los resultados de trabajos previos, entre ellos los de Leta y Chaimovich (2002), Leta, Glänzel y Thijs (2006) y Glänzel, Leta y Thijs (2006), que encontraron que Brasil pasó del $21.6 \%$ de colaboración en la década de los 80 al $26.7 \%$ en la década de los noventa. Por otra parte, Brasil mantiene el liderazgo en la productividad científica global de Latinoamérica, con un crecimiento anual del $8 \%$, aunque este crecimiento está por detrás de México (Glänzel, Leta y Thijs, 2006). Este liderazgo también se produce en algunas áreas específicas, como en Salud Pública, área en la que Brasil ocupa la primera posición en Latinomérica y la sexta mundial, seguido de México, Cuba, Colombia y Argentina (Zacca-González et al., 2014). 
El liderazgo de Brasil se explica por el hecho de que el gasto en investigación y desarrollo de este país representa el 60 \% de toda la región (Babini, 2011), lo que ha repercutido en que se convierta en el país que mayor colaboración internacional presenta, logrando un buen posicionamiento estratégico y un gran número de relaciones (Chinchilla-Rodríguez, Benavent-Pérez y Moya-Anegón, 2012). El crecimiento de Brasil también se ha observado en otras áreas, como en el campo de la Cerámica (Rojas-Sola y Jordá-Albiñana, 2009), Psicología (Sánchez-Sosa, 2008; Vera-Villarroel et al., 2011) o la Tecnología Sanitaria (Pichon-Riviere, Ceballos y Briones, 2009). Respecto a España, estudios previos han mostrado un crecimiento sostenido en los últimos años en diversas áreas, como la Neurología (González-Alcaide et al., 2008), la Bronconeumología (Granda Orive et al., 2009), la Cardiología (Aleixandre-Benavent et al., 2009) y la Pediatría (Alonso-Arroyo et al., 2013).

Entre los motivos del crecimiento científico de Brasil deben citarse el cese de la diáspora debido a las dictaduras, el incentivo de las agencias de fomento por una mayor velocidad de producción, lo que ha generado la necesidad de evaluar (Gracio y Oliveira, 2012); el aumento del número de estudiantes de educación superior y los nuevos planes para la incorporación de recursos humanos a las actividades científicas, sobre todo mediante la incorporación de programas de máster y doctorado, y programas específicos de introducción a la investigación como el Fellowship Initation in Science (Leta, Glänzel y Thijs, 2006), lo que ha llevado a una mayor cualificación del cuerpo de investigadores (Glänzel, Leta y Thijs, 2006). Otras razones para este crecimiento podrían estar en el aumento del número de revistas de América Latina incluidas en las bases de datos bibliográficas en la última década. Para la mayoría de los países, tales como Argentina, Chile, México y Venezuela, el número de revistas incluidas se ha multiplicado por tres, si bien el crecimiento ha sido más evidente en Brasil, donde el número de revistas se ha multiplicado por cuatro. Sin embargo, la cobertura de las revistas latinoamericanas en bases de datos bibliográficas internacionales sigue siendo baja y, en consecuencia, los trabajos de alta calidad procedentes de países de América Latina se publican por lo general en revistas de los Estados Unidos o en revistas europeas (Aleixandre et al., 2013).

A pesar de este crecimiento en el número de trabajos publicados, todavía perduran algunos problemas, como la inestabilidad en las dotaciones designadas a la investigación por parte de los organismos públicos. Así, el porcentaje del producto interior bruto (PIB) destinado a la investigación en Brasil, según datos facilitados por el Banco Mundial, fue en 2011 del 1.21 \%, más alto que el de otros países latinoamericanos como Chile (0.42\%), México $(0.43 \%)$ y Argentina $(0.65 \%)$, pero sensiblemente inferior al de la mayoría 
de los países más desarrollados. Este porcentaje fue del $1.36 \%$ en España, uno de los más bajos de la Unión Europea, ya que en países como Reino Unido es del 1.78 \% y del $2.89 \%$ en Alemania (World Bank, 2011). A pesar de lo expuesto, durante la década 1999-2008 España y Brasil fueron los países de la región iberoamericana que más aumentaron su inversión en $\mathrm{I}+\mathrm{D}$; asimismo, fueron los dos países iberoamericanos que presentaron el mayor número de investigadores, según el cómputo en Equivalencia a Jornada Completa (Albornoz, 2010).

La colaboración es fundamental para el desarrollo de la ciencia, ya que puede mejorar la eficiencia de los sistemas nacionales de $\mathrm{I}+\mathrm{D}+\mathrm{I}$ al permitir que los investigadores se integren en equipos internacionales, compartiendo recursos y aprendiendo nuevas técnicas (Cunningham y Dillon, 1997; Katz y Martin, 1997; Newman, 2004). El fenómeno de la colaboración es heterogéneo y ha sido expuesto por numerosos investigadores, como Beaver (2004), Newman (2004), Glänzel y Schubert (2001), entre otros. Los factores que más influyen en su génesis y mantenimiento son económicos, geopolíticos e intracientíficos (Glänzel y Schubert, 2001). La colaboración internacional puede reflejar tanto la movilidad individual o el interés de científicos individuales como la dependencia económica o política de un país o región, pero también la necesidad de tener acceso a equipos especiales que se comparten en proyectos multinacionales. En ciencias de la salud puede deberse incluso a factores biológicos, como la alta prevalencia de determinadas enfermedades en un país. La creación de redes y grupos que reúnen a científicos y tecnólogos de diferentes países es un aspecto central de las estrategias de cooperación, ya que permiten el avance del conocimiento, la mejora de la calidad y el aumento de la innovación y la competitividad (Cunningham y Dillon, 1997; Aleixandre et al., 2013). Por otro lado, la colaboración internacional es una señal positiva que indica la apertura a la investigación extranjera. Se ha reportado, además, que la investigación colaborativa produce unos resultados significativamente de mayor autoridad, lo que se refleja en el reconocimiento a partir de las citas, y una influencia de mayor duración, lo que se observa en la vida de las citas (Beaver, 2004).

El estudio previo de Mugnaini et al. (2014), que analizaba la colaboración bilateral Brasil-España por áreas temáticas, evidenció que dicha colaboración era más intensa en el área de Medicina Clínica y Experimental, siguiendo la clasificación propuesta por Glänzel y Schubert (2003), que agrupa las áreas Web of Science en 15 grandes categorías. La colaboración de Brasil se llevó a cabo especialmente con la Unión Europea, América Central y los Estados Unidos, que acaparó el 40.5 \% de la colaboración internacional. También se ha intensificado con países de la región, entre ellos México y Argen- 
tina, en consonancia con lo que mostraba en el estudio previo de Glänzel, Leta y Thijs (2006). Estos datos ratifican los resultados presentados por IRD e IEDCYT-CSIC (2009) relativos al periodo 2002-2006, que ya mostraban una amplia red de colaboración entre países de América Latina y Europa. Un estudio previo mostró que la mayor colaboración entre países dentro de la medicina clínica fue entre Brasil-Reino Unido, seguido de Brasil-Italia y Brasil-Francia. La colaboración con España ocupó el sexto puesto (De Filippo y Gómez, 2011). Según datos del Global Research Report para Brasil, elaborado por Thomson Reuters a partir de las bases de datos de la Web of Science (Adams y King, 2009), Estados Unidos es el principal colaborador de Brasil, seguido de Francia, Reino Unido, Alemania, Italia y Canadá. España ocupaba el séptimo lugar, pero dobló el número de artículos en colaboración del quinquenio 1998-2002 al 2003-2007, pasando de 1245 artículos en colaboración a 2 382, lo que supone el 19 \% del total de la producción de Brasil. En la base de este crecimiento deben situarse las políticas de fomento de la investigación y de la colaboración llevadas a cabo por los gobiernos de ambos países. La colaboración científica entre España y Brasil ha traído aparejada una amplia participación de otros investigadores procedentes de 121 países, especialmente Estados Unidos, seguido de países de la Unión Europea como Francia, Italia, Reino Unido, Alemania y Argentina, lo que es lógico y consecuencia de la política de la Unión Europea hacia la cohesión en la investigación de sus países miembros.

La capacidad científica de Brasil en el campo estudiado se concentra en ciertas instituciones, principalmente de los estados más ricos. Es evidente que en otros estados también debe existir investigación, pero ésta es más incipiente. Hacemos hincapié en el papel central de la Universidade de São Paulo en la red de colaboración científica y otras 10 instituciones brasileñas y 17 españolas que colaboran junto con instituciones de otros 16 países diferentes. Se pueden destacar instituciones como la Universidad de Buenos Aires, el Instituto Catalán de Oncología, el Hospital Clínic i Provincial de Barcelona, las universidades federales de São Paulo y de Rio de Janeiro y la Fundação Oswaldo Cruz.

\section{LIMITACIONES DEL ESTUDIO}

Este trabajo tiene algunas limitaciones que deben ser discutidas. En primer lugar, Scopus no incluye toda la literatura científico-médica publicada, por lo que otras bases de datos bibliográficas especializadas en recopilar revistas científicas de América Latina podrían haber sido utilizado de forma alterna- 
tiva o complementaria. Sin embargo, hemos utilizado Scopus porque tiene las siguientes ventajas: a) Es ampliamente utilizada en estudios que analizan la actividad científica, ya que incluye las principales revistas mundiales (Rojas-Sola y Jordá-Albiñana, 2009; Michán y Llorente-Bousquets, 2010); b) Proporciona los nombres de todas las afiliaciones institucionales que participan en los trabajos, lo que nos ha permitido determinar los indicadores de cooperación entre instituciones y países. En segundo lugar, este estudio no nos permite conocer la contribución al progreso científico de la colaboración científica entre Brasil y España porque no realiza un análisis conceptual de sus resultados; sin embargo, esta limitación puede ser considerada como una propuesta para futuras investigaciones.

\section{CONCLusiones}

Este estudio proporciona una indicación del estado de la colaboración entre Brasil y España en el campo de la Medicina con base en el análisis de los artículos publicados en revistas incluidas en la base de datos Scopus. Los resultados ponen de manifiesto el importante crecimiento en el número de artículos publicados en colaboración entre ambos países, la coparticipación de numerosos países de la Unión Europea, Latinoamérica y Estados Unidos, y que Brasil es una importante y creciente economía en investigación cuya capacidad en i+d crece rápidamente junto con México. La colaboración entre Latinoamérica y España debe continuar creciendo en términos de desarrollo intelectual y económico. Europa y España se han beneficiado del comercio de bienes con Latinoamérica en el pasado, por lo que hoy en día deben integrar sus conocimientos a través de la colaboración internacional, participando plenamente y evitando la marginación intelectual. Futuros trabajos en esta área podrían identificar los grupos de autores responsables de esta colaboración, así como las principales líneas de investigación entre ambos países.

\section{BiBLIOGRAFÍA}

Adams, J. y Ch. King. 2009. Global Research Report, Brazil. Research and collaboration in the new geography of science, June 2009. Leeds: Evidence Ltd.

Albornoz, M., dir. 2010. El estado de la ciencia: principales indicadores de Ciencia y Tecnología Iberoamericanos / Interamericanos. Argentina: REDES - Centro de Estudios sobre Ciencia, Desarrollo y Educación Superior. 
Aleixandre, J. A., E. Bordeu, J. L. Aleixandre-Tudó, M. Bolaños y R. Aleixandre-Benavent. 2013. "Scientific productivity and collaboration in viticulture and enology in Latin American countries". Ciencia e Investigación Agraria 40 (2): 429-443.

Aleixandre-Benavent, R., A. Alonso-Arroyo, F. J. Chorro-Gascó, F. Alfonso-Manterola, G. González-Alcaide, M. J. Salvador-Taboada et al. 2009. "La producción científica cardiovascular en España y en el contexto europeo y mundial (2003-2007)". Revista Española de Cardiología 62 (12): 1404-1417.

Alonso-Arroyo, A., J. González de Dios, M. Bolaños Pizarro, L. Castelló Cogollos, G. González Alcaide, C. Navarro Molina et al. 2013. "Análisis de la productividad e impacto científico de la pediatría española (2006-2010)”. Anales de Pediatría 78 (6): 409.e1409.e17.

Babini, D. 2011. "Acceso abierto a la producción científica de América Latina y el Caribe”. Revista Iberoamericana de Ciencia, Tecnología y Sociedad 6 (17): 1-24.

Balancieri, R., A. Botelho Bovo, V. Medina Kern, R. C. Santos Pacheco y R. Miranda Barcia. 2005. "A análise de redes de colaboração científica sob as novas tecnologias da informação e comunicação: um estudo na Plataforma Lattes". Ciência da Informação 34 (1): 64-77.

Batagelj, V. y A. Mrvar. 2008. "Pajek. Program for Large Network Analysis. Slovenia". University of Ljubljana. Disponible en: http:// vlado.fmf.uni-lj.si/pub/networks/pajek/ [Fecha de consulta: 20 de junio de 2013].

Beaver, D. 2004. "Does collaborative research have greater epistemic authority?" Scientometrics 60 (3):399-408.

Callon, M., J. P. Courtial y H. Penan. 1995. Cienciometría: la medición de la actividad científica: de la bibliometría a la vigilancia tecnológica. Asturias: Trea.

Chinchilla-Rodríguez, Z., M. Benavent-Pérez y F. Moya-Anegón. 2012. "International Collaboration in Medical Research in Latin America and the Caribbean (2003-2007)". Journal of the American Society for Information Science and Technology 63 (11): 2223 2238.

Cunningham, S. J. y S. M. Dillon. 1997. "Authorship patterns in information systems”. Scientometrics 39 (1): 19-27.

De Filippo, D. e I. Gómez. 2011. "Influencia de la colaboración internacional para los países latinoamericanos. Análisis de la cooperación España-Latinoamérica por área temática (WoS 2002-2006)”. En M. Albornoz y L. Plaza, ed., Agenda 2011: temas de indicadores de ciencia y tecnología. Buenos Aires: Red Iberoamericana de indicadores de Ciencia y Tecnología, 243-260.

Elsevier B. V. SciVerse Scopus. 2014. Base de datos. Disponible en: http://www.scopus.com/home.url [Fecha de consulta: 29 de abril de 2014]. 
FAPESP. 2011. Fundação de Amparo à Pesquisa do Estado de São Paulo. Relatório de atividades científicas 2010. Brasil: Produção Editorial da Gerência de Comunicação da FAPESP.

Glänzel, W. 2003. Bibliometrics as a research field: a course on theory and application of bibliometric indicators. Belgium: Katholieke Universiteit Leuven.

Glänzel, W. y C. A. Lange. 2002. "Distributional approach to multinationality measures of international scientific collaboration". Scientometrics 54 (1): 75-89.

Glänzel, W. y A. Schubert. 2001. "Double effort = Double impact? A critical view at international co-authorship in chemistry". Scientometrics 50 (2): 199-214.

_ y A. Schubert. 2003. "A new classification scheme of science fields and subfields designed for scientometric evaluation purposes". Scientometrics 56 (3): 357-367.

Glänzel, W., J. Leta y B. Thijs. 2006. "Science in Brazil. Part 1: A macro-level comparative study”. Scientometrics 67 (1): 67-86.

González-Alcaide, G., A. Alonso-Arroyo, J. González de Dios, A. P. Sempere, J. C. Valderrama-Zurián y R. Aleixandre-Benavent. 2008. "Redes de coautoría y colaboración institucional en Revista de Neurología”. Revista de Neurología 46 (11): 642-651.

Gracio, M. C. C. y E. F. T. Oliveira. 2012. "A inserção e o impacto internacional da pesquisa brasileira em "estudos métricos”: uma análise na base Scopus". Tendências da Pesquisa Brasileira em Ciência da Informação 5 (1): 1-19.

Granda Orive, J. I., S. Villanueva Serrano, R. Aleixandre-Benavent, J.C. Valderrama-Zurían, A. Alonso-Arroyo y F. García Río. 2009. "Redes de colaboración científica internacional en tabaquismo. Análisis de co-autorías a través del Science Citation Index durante el período 1999-2003". Gaceta Sanitaria 23: 222.e34-222.e43.

IRD e IEDCYT-CSIC. 2009. Coordinating the European Union and Latin American research and Innovation Networks. Deliverable 1.2. Report on indicators of bi-regional SET activities February 2009. Disponible en: http://s3.amazonaws.com/zanran_storage/www.s2lat. eu/ContentPages/44567013.pdf. [Fecha de consulta: 4 de abril de 2016].

Iribarren-Maestro, I., M. L. Lascurain-Sánchez y E. Sanz-Casado. 2009. "Are multi-authorship and visibility related? Study of ten research areas at Carlos III University of Madrid". Scientometrics 79 (1): 191-200.

Katz, J. S. y B. R. Martin. 1997. "What is research collaboration?” Research Policy 26: 1-18.

Leta, J. y H. Chaimovich. 2002. "Recognition and international collaboration: the Brazilian case". Scientometrics 53 (2): 325-335.

Leta, J., W. Glänzel y B. Thijs. 2006. "Science in Brazil. Part 2: Sectoral and institutional research profiles”. Scientometrics 67 (1): 87105. 
Michán, L. y J. Llorente-Bousquets. 2010. “Análisis bibliométrico de la sistemática biológica sobre América Latina durante el siglo XX en tres bases de datos mundiales". Revista de Biología Tropical 58 (2): 531-545.

Moya-Anegón, F., dir. 2013. Indicadores bibliométricos de la actividad cientifica Española 2010: publicación 2013. Madrid: Fundación Española para la Ciencia y la Tecnología (FECyT).

Mugnaini, R., D. De Filippo, S. A. Souza Vanz, S. Marugán, I. D. Chittó Stumpf y E. Sanz Casado. 2014. "Representatividade da colaboração entre Brasil e Espanha na Web of Science (2006-2012)". EBBC Encontro Brasileiro de Bibliometria o Cientometria, Recife.

Narin, F., D. Olivastro y K. S. Stevens. 1994. "Bibliometric theory, practice and problem”. Evaluation Review 18 (1): 65-76.

Newman, M. E. J. 2004. "Coauthorship networks and patterns of scientific collaboration". Proceedings of the National Academy of Sciences of the United States of America 101 (1): 5200-5205.

Okubo, Y. 1997. Bibliometric indicators and analysis of research systems: methods and examples, Paris: OECD.

Olmeda-Gómez, C., A. Perianes-Rodríguez y M. A. Ovalle-Perandones. 2008. "Estructura de las redes de colaboración científica entre las universidades españolas". Ibersid 3: 129-140.

Otte. E. y R. Roussesu. 2002. "Social network analysis: a powerful strategy, also for the information sciences". Journal of Information Science 28 (6): 441-453.

Persson, O., W. Glänzel y R. Dannell. 2004. "Inflationary bibliometric values: the role of scientific collaboration and the need for relative indicators in evaluative studies". Scientometrics 60 (3): 421-432.

Pichon-Riviere, A., R. M. Ceballos y E. Briones. 2009. "Health technology assessment in Latin-america and the Caribbean, facilitators and barriers for international collaboration: a survey". Value in Health 12 (7): A488.

Rojas-Sola, J. I. y B. Jordá-Albiñana. 2009. “Análisis bibliométrico de las publicaciones científicas españolas en la categoría materials science, ceramics de la base de datos JCR (SCI) (1997-2008)". Boletín de la Sociedad Española de Cerámica y Vidrio 48 (5): 255-260.

Sánchez Sosa, J. J. 2008. "Professional collaboration between psychologists and other health professionals in healthcare settings in Latin America”. International Journal of Psychology 43: 171.

SCImago Lab. 2013. SCImago Journal \& Country Rank (SJR). Disponible en: http://www.scimagojr.com [Fecha de consulta: 08 de abril de 2013].

Spinak, E. 1998. "Indicadores cienciométricos". Ciência da Informação 27 (2): 141-48.

Vera-Villarroel, P., W. López-López, S. Lillo y L. Silva. 2011. "La producción científica en psicología latinoamericana: un análisis de la investigación por países". Revista Latinoamericana de Psicología 43 (1): 95-104. 
Wasserman, S. y K. Faust. 1994. Social network analysis: methods and applications. Cambridge: Cambridge University Press.

World Bank. 2011. Data Word Bank. Disponible en: http://data.world bank.org/indicator/NY.GDP.MKTP.CD [Fecha de consulta: 15 de noviembre de 2014].

Zacca-González, G., Z. Chinchilla-Rodríguez, B. Vargas-Quesada y F. Moya-Anegón. 2014. "Bibliometric analysis of regional Latin America's scientific output in Public Health through SCImago Journal \& Country Rank”. BMC Public Health 14: 632.

Para citar este artículo:

Alonso Arroyo, Adolfo et al. 2016. "Un análisis bibliométrico en el área de la Medicina: colaboración científica entre Brasil y España (2002-2011)." Investigación Bibliotecológica: Archivonomía, Bibliotecología e Información 69: 205-229. http://dx.doi.org/10.1016/j.ib bai.2016.04.018 
